\title{
On the Oxidation Behavior of Titanium within Coated Nickel-Based Superalloys
}

\author{
R.J. Bennett ${ }^{a^{*}}$, R. Krakow ${ }^{a}$, A.S. Eggeman ${ }^{a}$, C.N. Jones ${ }^{b}$, H. Murakami ${ }^{c}$, C.M.F. Rae ${ }^{a}$ \\ ${ }^{\text {a }}$ Rolls-Royce UTC, Department of Materials Science and Metallurgy, University of \\ Cambridge, 27 Charles Babbage Road, Cambridge, CB3 OFS, United Kingdom \\ ${ }^{\mathrm{b}}$ Rolls-Royce plc, P.O. BOX 31, Derby, DE24 8BJ, United Kingdom \\ ${ }^{\mathrm{c}}$ National Institute for Materials Science, 1-2-1 Sengen, Tsukuba, Ibaraki, 305-0047 Japan
}

\begin{abstract}
Rutile precipitation within alumina scales grown on coated nickel based superalloy CMSX-4 has been found to occur preferentially at grain boundaries within the scale. Misorientation analysis using Rodrigues-Frank space has revealed clustering of the misorientation between neighbouring grains of corundum and rutile about the established $<0001>_{C}\{11 \overline{2} 0\}_{C} / /$ $<010>_{R}\{101\}_{R}$ orientation relationship observed in Ti-containing sapphire crystals. The fraction of interfaces found to exist in this configuration is sufficient to explain the nucleation of rutile from a single corundum grain abutting the rutile grain. The diffusive behaviour of Ti has been observed to vary considerably within three commercially used coatings, a plain aluminide coating, a plat-aluminide coating and a diffused platinum coating. Titanium diffusion is enhanced by the presence of Pt. However this did not lead to the precipitation of more rutile, which although observed in all three coatings, was present in sufficient quantity to be detected using XRD only within the plain aluminide coated samples.
\end{abstract}

Keywords: Nickel-based superalloy; Coating; Oxidation; Thermal Barrier Coatings (TBC);

EBSD; Orientation Relationship

* Corresponding Author. E-mail address: rjb222@cam.ac.uk Phone: +44 (0) 1223334434

\section{Introduction}


In recent years, the increased temperature capability of nickel-based superalloys used in gas turbine applications has been largely due to the application and improvement of thermal barrier coatings (TBCs) and associated gas cooling. Current combustion temperatures in commercial gas turbines make thermal barrier coatings a necessity for both turbine blades and static vanes operating in the first stage of the turbine. Thus increasing the longevity of these coatings, limited by TBC spallation, is of the upmost importance to the industry. One such major improvement has been the electro-deposition of a thin Pt layer to the substrate as a stand-alone bond layer under the YSZ [1,2]. Spallation tests for superalloys coated with this diffused Pt coating (also termed Pt-modified or duplex $\gamma / \gamma^{\prime}$ coating), consistently display very good TBC spallation life compared with Pt-aluminide, plain aluminide and MCrAlY bond coats, with results consistent over a number of substrates $[3,4,5]$.

In the last five years however, there has been increasing evidence that the use of Pt within coatings alters the activity of $\mathrm{Ti}[5,6]$, a low density alloying element, used for stabilizing and strengthening $\gamma^{\prime}$. It is well established that Pt decreases the activity of Al, which then results in the uphill diffusion of $\mathrm{Al}$ from the substrate, maintaining a high concentration close to the surface facilitating alumina scale growth and re-formation. Pint et al. [5] have obtained micro-probe measurements showing raised Ti concentrations close to the surface of Diffused-Pt coated CMSX-4 after prolonged thermal cycling. Tawancy et al. [6] observed uphill Ti diffusion for the same system, with Ti and Pt both segregating to the $\gamma^{\prime}$ phase. Ab-initio calculations using density functional theory have shown that Pt stabilizes defect formation in $\beta$-NiAl, enhancing anti-site assisted and solute vacancy diffusion mechanisms $[7,8]$.

Titanium (IV) oxide, exists as a multitude of polymorphs, three of which are stable at atmospheric pressure; anatase, brookite and rutile. Of these, brookite is difficult to synthesise and literature on its formation is sparse. The phase transformations between anatase and rutile have been extensively reviewed by Hanaor et al. [9]: anatase transforms to the stable rutile structure in air, at temperatures far below those experienced in gas turbines, although reported transformation temperatures do vary considerably $[10,11]$. Upon the application of pressure, 
the tetragonal rutile structure has been found to undergo a transformation to an orthorhombic polymorph with a $\alpha-\mathrm{PbO}_{2}$ structure, known as $\alpha-\mathrm{TiO}_{2}[12]$. At temperatures below $500^{\circ} \mathrm{C}$, this critical pressure falls within the realm of TGO stresses measured during thermal cycling [13]. The $\alpha-\mathrm{TiO}_{2}$ phase has been observed to transform into rutile following stress relaxation within Ti doped sapphire [14].

The solubility of $\mathrm{Ti}$ within alumina scales is thought to occur via the formation of $\mathrm{Ti}_{\mathrm{Al}}$ substitutional defects for $\mathrm{Ti}^{3+}$ ions and vacancy clusters consisting of $3 \mathrm{Ti}^{\circ}{ }_{\mathrm{Al}}$ and $\mathrm{V}^{\prime \prime \prime}{ }_{\mathrm{Al}}$ for $\mathrm{Ti}^{4+}$, preserving charge neutrality within the scale in accordance with the Schottky formalism [15] [16]. If a divalent cation $\mathrm{M}$ is available, $\mathrm{Ti}^{\circ}{ }_{\mathrm{Al}} \mathrm{M}_{\mathrm{Al}}^{\prime}$ pairs may form, a theory supported by defect calculations performed by Grimes [17], although this has only been verified for $\mathrm{Mg}$. Measurements of lattice parameter changes within Ti-doped sapphire by Mckee and Aleshin [18] and Roy and Coble [19] have indicated lower solubility of $\mathrm{Ti}^{4+}$ than $\mathrm{Ti}^{3+}$ within $\alpha-\mathrm{Al}_{2} \mathrm{O}_{3}$ for annealing temperatures in excess of $1250^{\circ} \mathrm{C}$ in air and $1400^{\circ} \mathrm{C}$ in $\mathrm{N}_{2}$ (firing in nitrogen reduces the $\mathrm{Ti}^{4+}$ ions to $\mathrm{Ti}^{3+}$ above this temperature). Above the solubility limit, rutile $\mathrm{TiO}_{2}$ forms 'needle' and 'heart-shaped' precipitates, which are usually twinned [20,21]. At 1200$1300^{\circ} \mathrm{C}, \mathrm{He}$ et al. [21] observed $\alpha-\mathrm{TiO}_{2}$ precipitates which form initially, subsequently undergo twinning to minimize coherency strain energy with the sapphire matrix, then transform to the rutile structure. The long axis of the rutile needles, [011] ], lies parallel to the $<10 \overline{1} 0>$ directions in $\alpha-\mathrm{Al}_{2} \mathrm{O}_{3}$. This preferred orientation relationship (OR) between the two phases, leads to the 'asterism' seen in star sapphires. This OR has also been observed more recently Daneu et al. [22], the registry is typically defined as $[0001]_{C}(11 \overline{2} 0)_{C} / /[010]_{R}(101)_{R}$. Langensiepen et al. [23] observed the precipitation of $\mathrm{Al}_{2} \mathrm{TiO}_{5}$ above 0.6 cation $\%$ titanium at triple junctions within polycrystalline sapphire. However, Within the $\mathrm{Al}_{2} \mathrm{O}_{3}-\mathrm{TiO}_{2}$ binary system Slepetys et al. [24] have found the $\mathrm{Al}_{2} \mathrm{TiO}_{5}$ to be unstable below about $1240^{\circ} \mathrm{C}$ during heat treatment in oxygen. 
Tawancy et al. [6] concluded that the formation of $\mathrm{TiO}_{2}$ within scales formed on diffused Pt coated nickel superalloys, leads to increased oxide spallation. This is thought to occur via de-lamination of the alumina scale, assisted by void formation at $\mathrm{TiO}_{2}$ particles forming at the oxide/metal interface. Other recent investigations of the resistance of these coatings to high temperature thermal exposure $[25,26]$ have consistently observed rutile growth on plain aluminide coatings. Shirvani et al. [26] observed $\mathrm{TiO}_{2}$ rutile formation upon plain aluminide coated CMSX-4 but not upon Pt-aluminide coated sample after cyclic oxidation.

The progressive removal of titanium from alloys designed for optimum environmental resistance has been a strong trend for a number of years, but our current understanding of the role Ti plays in such degradation of thermal barrier coatings is limited. As yet there has been no comparative study between the effects of different coating composition on the diffusional and oxidative behavior of Ti. The orientation relationship between rutile and corundum is well characterized in Ti-doped sapphire, but as yet there is no evidence for any orientation relationships occurring within thermally grown alumina scales. The main purpose of this investigation is therefore to observe and characterize the behavior of $\mathrm{Ti}$ and associated rutile formation within several bond coat systems during high temperature oxidation. Knowledge of the precipitation mechanism will assist in our understanding of the stress distributions associated with rutile formation in oxide scales.

\section{Experimental}

For this investigation, three commercially used diffusion coatings were applied to single crystal CMSX $-4{ }^{\circledR 1}$. Cylindrical test bars were cast by Rolls Royce as single crystals with the $<001>$ orientation aligned with the cylinders long axis. Bars were then subjected to hot isostatic pressing to reduce porosity and solutioning and aging heat treatments, forming cuboidal $\gamma^{\prime} \mathrm{Ni}_{3} \mathrm{Al}$ precipitates approximately $0.5 \mu \mathrm{m}$ in dimension to replicate the microstructure of components in service.

\footnotetext{
${ }^{1} \mathrm{CMSX}^{\circledast}$ and CMSX-4 ${ }^{\circledast}$ are registered trademarks of Cannon-Muskegon Corporation.
} 
Samples were coated at Turbine Surface Technologies Limited (TSTL) in Annesley, Nottinghamshire, UK. Following normal commercial protocols, Pt was electro-deposited to a thickness of $7 \mu \mathrm{m}$ in the Pt-aluminide samples and $10 \mu \mathrm{m}$ to form the diffused Pt coating. In each case the samples were then annealed for one hour at $1100^{\circ} \mathrm{C}$ and $1150^{\circ} \mathrm{C}$ for each coating respectively. Vapor phase aluminizing was used to produce high temperature low activity (HTLA) aluminide and Pt-aluminide coating microstructures. The resulting microstructures within each coating in the as received condition are shown in figure 1 . The presence of both $(\mathrm{Ni}, \mathrm{Pt}) \mathrm{Al}$ and $\mathrm{PtAl}_{2}$ phases within the Pt-aluminide coating is similar to that of an intermediate stage between outward and inwardly grown coating microstructures [27]. This is partly due to the slightly lowered temperature used during aluminizing and thicker Pt layer, reducing outward nickel diffusion. As the aluminium chips are pre-alloyed the designation 'Low-Activity' is still used.

Samples of coated CMSX-4 were oxidized both isothermally and cyclically in air at $1100^{\circ} \mathrm{C}$. The cycle consisted of a 5-minute ramping period, followed by a 55-minute hold at temperature, before a 20-minute cooling period. After 1, 25 and 100 cycles, oxide and coating phases were analyzed using XRD. Samples were oxidized isothermally for 25, 100 and 500 hours. A JOEL 5800 scanning electron microscope was used to obtain micrographs. The diffusion of alloying elements within each coating has been mapped and quantified using Wavelength Dispersive X-ray Spectroscopy (WDS) on a Cameca SX100 electron microprobe. Glow Discharge Optical Emission Spectroscopy (GD-OES) measurements were obtained using a Horiba GD-profiler 2 spectrometer, while XRD data were obtained using a Rint-2500 X-ray diffractometer. Transmission electron microscopy EDS data was collected using a JOEL JEM ARM-200F transmission electron microscope at $200 \mathrm{keV}$. The diffraction patterns, used for phase identification and orientation mapping, were recorded at $300 \mathrm{keV}$ on a CM300 FEG-TEM that was retro-fitted with a Nanomegas ASTAR unit. This allows fast scanning of the electron beam across the sample with a high-rate digital camera used to record a diffraction pattern at each point of the scan [28]. The digitized pattern is compared to templates determined from the expected crystal structure(s) of the sample. Pattern matching is 
performed using a cross-correlation calculation (or correlation index) between the experimental pattern and the template patterns. The reliability of the pattern match is done by comparing the correlation index for the best pattern match with the index score for the second best match in the library. Large reliability therefore indicates an unambiguous orientation determination. This comparison allows the phase and orientation of each pixel in the scan to be determined.

The ASTAR system was used to study the orientation of a rutile particle $\left(\mathrm{TiO}_{2}\right.$, tetragonal, space group $\left.\mathrm{P} 4_{2} / \mathrm{mnm}\right)$ and two surrounding corundum grains $\left(\mathrm{Al}_{2} \mathrm{O}_{3}\right.$, trigonal, space group $R \overline{3} c$ ) in a region of interest approximately $750 \times 350 \mathrm{~nm}^{2}$. A cropped image is depicted in figure 7 . The camera length used was $17 \mathrm{~cm}$ and the spot size and step size in either direction were $5 \mathrm{~nm}$. For this data set precession electron diffraction was not utilized. Plotting and grain reconstruction were performed using the Matlab toolbox - Mtex [29]. For EBSD analysis, an FEI Nova (FEG-SEM) attached to a Bruker EBSD acquisition system was used. Data cleaning and grain reconstruction was performed within HKL Channel 5 software and subsequent disorientation analysis was performed using code written in the Matlab programming language. The 'HKL' definition of hexagonal axes where $\mathrm{x} / /[10 \overline{1} 0]$ and $\mathrm{y} / /$ [12 10$]$ has been applied for misorientation analysis. It should be noted that ASTAR assigns orientation to indexed patterns according to the alternative 'TSL' hexagonal axes convention. When EBSD and ASTAR data are plotted on the same set of axes, a transformation matrix, corresponding to a $30^{\circ}$ rotation about the z-axis was applied to the corundum data obtained using ASTAR.

\section{Results}

\subsection{Coating Microstructural Evolution}

The backscattered micrographs of the three coatings are shown in figure 1 prior to oxidation (a-c) and after 100 hours at $1100^{\circ} \mathrm{C}(\mathrm{d}-\mathrm{f})$. The concentration profiles for the major elements, measured using WDS, are shown in figure 2. The sputtered region using GD-OES is of the order of $1 \mathrm{~mm}$, so is less sensitive to local variations in chemical composition, but 
gives a more reliable absolute value for the composition and is a useful comparison for the WDS results. The results from the two techniques are compared for the same samples in figure 2e and similar depth profiles for $\mathrm{Al}, \mathrm{Co}, \mathrm{Pt}$ and Ni can clearly be seen. X-ray diffraction data showing the coating phase composition close to the surface from each sample is shown in figure 3.

From figures $2 \mathrm{a}, 2 \mathrm{~b} 3 \mathrm{a}$ and $3 \mathrm{~b}$, the plain aluminide coatings initially show an almost 1:1 $\mathrm{Ni}: \mathrm{Al}$ ratio and a $\beta$-NiAl single phase microstructure that transforms to a mixture of Ni-rich $\beta$ and $\gamma^{\prime}$. The $\gamma^{\prime}$ forms wherever the Al flux away from the coating is greatest, initially in the inter-diffusion zone and in isolated patches at the coating/oxide interface. After 500 hours, the coating is mostly $\gamma^{\prime}$, as shown in figure $3 \mathrm{a}$.

The initial Pt-aluminide coating consists of a four-layered microstructure defined by the four principal phases shown in figures $3 \mathrm{c}$ and $3 \mathrm{~d}$. It consists of principally $\xi-\mathrm{PtAl}_{2}$ at the surface, atop a mixture of $\beta-\mathrm{Ni}(\mathrm{Pt}) \mathrm{Al}$ and $\xi$. Below this is an outer diffusion zone of $\beta$, TCPs and inner diffusion zone of TCP, $\gamma$ and $\gamma^{\prime}$. After prolonged exposure, the $\mathrm{PtAl}_{2}$ is destabilized through inward Pt and $\mathrm{Al}$ diffusion, as well as outward Ni diffusion into the coating and consumption of $\mathrm{Al}$ through oxide formation, as shown by a decrease in $\mathrm{Al}$ and $\mathrm{Pt}$ and increase in $\mathrm{Ni}$ concentration from figure $2 \mathrm{c}$ to $2 \mathrm{~d}$. This loss induces a transformation to single phase $\beta$ $\mathrm{Ni}(\mathrm{Pt}) \mathrm{Al}$ occurs. The $\beta$ phase remains far more stable than in the plain aluminide coating, as even after 500 hours, very little $\beta$ to $\gamma^{\prime}$ transformation has occurred. The coating thickness also doubles during this exposure.

The diffused Pt coating exhibits very high $\gamma^{\prime}$ volume fractions prior to oxidation, indicated by superlattice reflections in figures $3 \mathrm{e}-\mathrm{f}$ and enhanced backscattered contrast in figure $1 \mathrm{c}$ due to Pt segregating preferentially to $\gamma^{\prime}$. The volume fraction of $\gamma$ at the coating surface increases during exposure, shown in both figures $3 \mathrm{e}$ for isothermal and $3 \mathrm{f}$ for cyclic oxidation, and $\mathrm{Al}$ concentrations, depicted in figure $2 \mathrm{f}$ stabilize at a level just above that in the bulk ( $\sim 10$ at.\%). After 1 hour at $1100^{\circ} \mathrm{C}$, the oxide consists primarily of $\alpha-\mathrm{Al}_{2} \mathrm{O}_{3}$ with no transient alumina polymorph $(\gamma, \theta)$ detected using XRD. After prolonged exposure the 
formation of $\mathrm{NiAl}_{2} \mathrm{O}_{4}$ spinel is detectable in the Pt-aluminide and diffused Pt samples, while rutile $\mathrm{TiO}_{2}$ is also detected within the plain aluminide coated samples.

\subsection{Ti diffusion and Oxide formation}

The diffusion profiles for minor elements have also been measured using WDS and are shown in figure 4 . The uphill diffusion of Ti can clearly be seen in the diffused Pt coating, but not in either of the aluminized coatings. Significant Ti concentrations within this duplex $\gamma / \gamma^{\prime}$ coating are observed prior to thermal exposure, indicating that Ti is highly mobile at the low oxygen partial pressures used during annealing. As with all major elements, GD-OES measurements for Ti and Mo depth profiles were obtained, for the as-received diffused $\mathrm{Pt}$ coated sample. The results of GD-OES also show that the Ti concentration is enriched at the coating surface. Error bars are shown for the WDS data for elements (Hf and Mo), the standard deviation of which exceeds the marker size.

Regions of each sample, after 100 hours isothermal exposure, were mapped with WDS close to the surface scale and these maps are shown in figure 5. In all three coated samples, Ti 'clusters' within the oxide scale can clearly be observed. Initially it was thought that a lack of oxide spallation in the Pt containing coatings could attenuate scattered x-ray intensity received from the rutile, hiding the peaks, particularly at low grazing angle. Therefore samples were thermally cycled to elucidate whether this effect was indeed occurring. However it can clearly be seen in figure 3 that the difference between cycled samples (right hand side) and isothermal (left) is minimal; there is no significant change in oxide phase composition from one type of test to the other.

Foils from each coating were prepared, using the FIB lift out technique. The samples were oxidized for 25 hours at $1100^{\circ} \mathrm{C}$ to ensure little or no spallation had occurred. Energy dispersive $\mathrm{x}$-ray spectroscopic mapping, shown in figure 6 , reveals Ti concentrations in two isolated regions of the oxide scale grown on the diffused Pt coated TEM foil. Subsequent diffraction pattern analysis reveals these particles have the rutile $\mathrm{P} 4_{2} / \mathrm{mnm}$ space group. 
In order to compare the orientation of the rutile particle with the surrounding alumina grains, the particle shown in figure 6 was mapped using ASTAR to observe possible orientation relationships with the surrounding $\alpha-\mathrm{Al}_{2} \mathrm{O}_{3}$. This was done at two tilts, in order to achieve zone axis diffraction patterns for both rutile and one of the two surrounding corundum grains. The indexation resulted in high values for the correlation index and reliability in the area of interest with the exception of grain and phase boundaries that usually have lower values of both due to overlapping diffraction patterns. The results of the mapping for one orientation are shown in figure 7.

For the grain reconstruction a threshold angle of $10^{\circ}$ was chosen, based on previous experience, to define individual grains. In the area of interest all grains with less than four measurements per grain have been filtered out to reduce the noise level. The remaining orientation map in figure $7 \mathrm{~b}$ shows three main areas next to an edge in the TEM foil. The blue and the purple regions represent two orientations of corundum grains, also annotated, whereas the burgundy area represents the orientation of a rutile grain. The orientations are also highlighted in the inverse pole figure for rutile (Figure 7c) and corundum (Figure 7d). When analyzing the disorientation between the corundum grains in detail a disorientation angle of $49.74^{\circ}$ can be found; there is no particular significance of this disorientation.

In order to observe the orientation relationship between rutile and corundum with some degree of statistical significance, two EBSD maps were obtained from regions of the TGO formed on a plain aluminide coated sample after 500 hours at $1100^{\circ} \mathrm{C}$. These maps are shown in figure 8, with EDS data acquired simultaneously. This EBSD data was then used for disorientation analysis between the two crystal structures, this procedure is briefly outlined as follows: The non-translational symmetry elements of the rutile and corundum structures are defined by the point group symmetries $4 / \mathrm{mmm}$ and $\overline{3} \mathrm{~m}$ respectively. The misorientation matrices $m_{R C}$ between all adjacent grains of rutile $(\mathrm{R})$ and corundum $(\mathrm{C})$ were studied in order to observe any orientation relationship between them. The calculation of $m$, requires the use of symmetry operators (denoted as $O$ ), corresponding the to the $4 / \mathrm{mmm}$ and $\overline{3} \mathrm{~m}$ point groups. 


$$
m_{R C}=O_{C} g_{C}\left(O_{R} g_{R}\right)^{-1}(1)
$$

The Rodrigues-Frank (RF) representation of both grain orientations and disorientation between neighboring grains was proposed by Heinz and Neumann [30] for disorientation analysis. Rodrigues-Frank space is suitable for visualizing the combined symmetry for two point groups on one plot. He and Jonas [31] have explored the shapes of these domains within RF space for different Laue groups.

The RF vector is calculated from an orientation matrix for any given grain and constitutes an angle of rotation $\omega$ along with a corresponding rotation axis $\underline{n}$. This angle/axis pair can be calculated as the eigenvalue and eigenvector respectively of orientation and misorientation matrices. All symmetrically equivalent orientation matrices are considered and the one(s) that fulfill(s) the criterion of having the smallest rotation angle $\omega$ are represented. For a given disorientation, $m, \omega$ and $\underline{n}$ are defined as a rotation between orthogonal axes within both crystal systems.

$$
\underline{r}=\tan \left(\frac{\omega}{2}\right) \cdot \underline{n}
$$

The Rodrigues-Frank vector $\underline{r}$ is truncated by the tangent of the angle $\omega$. This yields an important result, namely that the domain shape for all $\underline{r}$ that is formed either analytically or through Monte-Carlo simulations (as in this investigation), is a representation of the crystal point group symmetry. In a trigonal crystal (point group $\overline{3} \mathrm{~m}$ ) this is a 'squashed' six-sided polygon, in a tetragonal crystal (point group $4 / \mathrm{mmm}$ ) the domain is a polygon with eight sides. The maximum symmetrized rotation angle that can exist purely about the $\mathrm{c}$ (parallel to z) axis is 60 degrees in the corundum structure and 45 degrees in the rutile, leading to a decreased height of the $\overline{3} \mathrm{~m}$ and $4 / \mathrm{mmm}$ RF space to $\tan (\pi / 6)$ and $\tan (\pi / 8)$ respectively. The Rodrigues-Frank vectors within RF space for corundum and rutile nearest neighbor grains are shown in figures $9 a$ and $9 b$. The disorientation space is shown in figure $9 c$ and corresponding disorientation angle distribution in $9 \mathrm{~d}$. Two clear clusters can be seen centered very close to the $[0,1,0]$ and $[0,-1,0]$ Cartesian axes within figure $9 \mathrm{c}$. The angle of rotation is close to $90^{\circ}$. 
The Rodrigues-Frank vectors corresponding to the minimum angle of rotation for the ASTAR mapped grains are also plotted for comparison.

\section{Discussion}

\subsection{Coating Degradation}

Three commercially used thermal barrier coating systems, consisting of a diffusion coating applied to a second generation nickel based superalloy have been exposed both isothermally and cyclically at $1100^{\circ} \mathrm{C}$ in air, to examine the effects of thermal exposure on micro-structural evolution, oxide formation and diffusion kinetics. The precipitation of $\gamma^{\prime}$ within single phase $\beta-\mathrm{Ni}(\mathrm{Pt}) \mathrm{Al}$ is retarded by the presence of $\mathrm{Pt}$, due to the maintenance of a slightly higher $\mathrm{Al}$ concentration within the coating. The transformation from $\xi-\mathrm{PtAl}_{2}+\beta-$ $\mathrm{Ni}(\mathrm{Pt}) \mathrm{Al}$ to pure $\beta$ was also found to require several cycles to occur at this temperature. The near surface microstructure of the diffused Pt coated samples were found to change from predominantly $\gamma^{\prime}$ to $\gamma$. The presence of Pt within the fcc and $\mathrm{L} 1_{2}$ phases can maintain a higher $\mathrm{Al}$ concentration, than in the bulk, however during exposure in air, a slight outward flux of Al (due to scale growth and re-formation) and dilution of Pt, substantiates this transformation from $\gamma^{\prime}$ to $\gamma$. No martensitic $\mathrm{L}_{0}$ phase was is believed to have formed within either the aluminide or Pt-aluminide coatings. This is most evident from the lack of any observable peak $\left(\mathrm{L1}_{0}(200)\right)$ close to 47 degrees two theta. This phase has been observed to form at temperatures below $620^{\circ} \mathrm{C}$ during high temperature in situ XRD measurements and after

oxidation at $1150^{\circ} \mathrm{C}$ within aluminized [32] and Pt-aluminized René N5 [33].

\subsection{Behavior of $\mathrm{Ti}$}

The superior detection limits of wavelength dispersive x-ray spectroscopy over the energy dispersive technique have enabled the accurate quantification of of Ti and Mo (Hf less so) within these coating systems. With a low free energy of oxide formation, it was anticipated that Ti would diffuse readily to the surface, and this is indeed the case. After prolonged exposure, the equilibrium concentrations of these elements increase significantly with the 
incorporation of $\mathrm{Pt}$ within the $\beta$-NiAl phase. This result is consistent with the theory proposed by Marino et al. $[7,8]$ that the Pt effect increases defect stability within $\beta$-NiAl, lowering the migration energy barrier for $\mathrm{Al}$ diffusion, and thereby also increasing the Ti equilibrium concentration within the B2 structure. The uphill diffusion observed within the diffused $\mathrm{Pt}$ coating also suggests that this effect is present within $\gamma^{\prime}$ when considering the work conducted by Tawancy et al. [6].

The XRD traces indicate much more rutile formation within the plain aluminide samples than either the Pt-aluminide or the diffused Pt coatings. This result is consistent with the findings of Shirvani et al. [26], who cyclically oxidized specimens of plain aluminide and Ptaluminide coated Rene-80 (containing 4 wt.\% Ti). The oxidation regime appears to have little effect on the nature of the oxide formed at $1100^{\circ} \mathrm{C}$, although accelerated precipitation of rutile is observed during cycling. The results of WDS mapping, reveal small Ti rich particles, within the $\alpha-\mathrm{Al}_{2} \mathrm{O}_{3}$ on all three types of coating after thermal exposure. These particles appear most abundant on the plain aluminide sample. Examination using TEM selected area diffraction and EBSD has confirmed that these precipitates are indeed rutile. While the location of these particles in WDS maps within the TGO is difficult to determine, close inspection of the $\mathrm{Ti}$ and $\mathrm{Al}$ maps in figure 5, reveals that the Ti rich particles are located away from the scale surface, within the plain aluminide coating. These precipitates are abundant and can be presumed to be the source of rutile peaks observed within XRD spectra. After 500 hours of isothermal oxidation, rutile particles within this coating are found to occur approximately half way through the scale, clearly seen within figure 8 , so the precipitates are encapsulated within the TGO. It has been hypothesized by Tawancy et al. [6] that void formation at the metal/oxide interface from $\mathrm{TiO}_{2}$ precipitates leads to increased scale spallation. The implication of our findings is that spallation resistance cannot be so obviously impaired by rutile precipitation as concluded by Tawancy et al. [6], as the scale does not always spall and continues to grow beneath the precipitates. The scale can accommodate a large volume fraction of these precipitates, which is clear from figure 8 . The evolution of the stress/strain relationship between matrix and an embedded particle would be useful to 
ascertain how the scale can accommodate such a large fraction of rutile precipitates and remain intact.

\subsection{Orientation between rutile and corundum}

The orientation between a rutile particle and two surrounding corundum grains has been mapped using TEM retro-fitted with a Nanomegas ASTAR unit. Further analysis of the orientation of the two phases using stereographic projection, reveals that neither of the two alumina grains fulfills the orientation relationship with the rutile grain reported in the literature. This is also shown in Rodrigues-Frank space within figure 9c, along with EBSD data for direct comparison. Mapping of the TGO containing rutile particles using EBSD has revealed an orientation relationship between adjacent corundum (point group $\overline{3} \mathrm{~m}$ ) and rutile $(4 / \mathrm{mmm})$ phases. This orientation relationship is characterized by two clusters in figure $9 \mathrm{c}$, about the $[0,1,0]$ and $[0,-1,0]$ Cartesian rotation axes. From the disorientation angle distribution shown in figure $9 \mathrm{~d}$, approximately $1 / 4$ of the 284 interfaces between adjacent grains analyzed, are close to this specific misorientation angle. Despite the fact that this is clearly not random, a degree of non-randomness can be gained from knowledge of the random distribution of disorientation angles between the two structures. Following a procedure outlined by Morawiec [34,35] the density of disorientation angles between rutile and corundum has been calculated and is shown as a solid black line within figure $9 \mathrm{~d}$. Over $1 / 5$ of the total number of interfaces analyzed have a non-random misorientation at 90 degrees. The precipitation of rutile occurs preferentially at triple and quadruple junctions, nucleating from a single corundum grain, hence the expected fraction of nearest neighbor grains that will adopt the orientation relationship for nucleation is close to $1 / 4$. It is therefore entirely plausible that the vast majority of rutile grains observed precipitate from an adjacent corundum grain with the $<0001>_{C}\{11 \overline{2} 0\}_{C} / /<010>_{R}\{101\}_{R}$ orientation relationship reported for sapphire $[14,20,21]$. 
The mapping of the two structures onto one another using the positive y-axis as the axis of rotation is shown in figure 10. Both structures are shown in their reference orientations (corresponding to an orientation matrix equal to the identity matrix). A 90 degree rotation about the $[\overline{1} 2 \overline{1} 0] / / y$-axis in the corundum structure (as shown in 10a) brings the two planes (011) and ( $\overline{2} 110)$ close to parallel. The construction of the Rodrigues-Frank vector involves choosing the minimum angle of rotation and corresponding axis or axes for any given misorientation, thereby showing the disorientation between the grains. The exact orientation relationship is formed from a disorientation angle very close to the clusters of data shown in figure $9 \mathrm{c}$. This is given as a $90.034^{\circ}$ rotation about four different axes, which are shown in table 1 . This multiplicity of four arises from a rotation of $5.6^{\circ}$ about the $[100]_{R}$ axis between $(011)_{\mathrm{R}} / /(11 \overline{2} 0)_{\mathrm{C}}$ and $(01 \overline{1})_{\mathrm{R}} / /(\overline{2} 110)_{\mathrm{C}}$ and visa-versa. Two pairs of two rotation axes, clustered close to $[0,1,0]$ and $[0,1,0]$, are therefore equally probable depending on the indexation of the Kikuchi pattern. The orientation relationship within RF space is then defined as a disorientation angle and axis by not one but four Rodrigues Vectors, each with the same minimum angle of rotation. The clustering of disorientations about these four axes represents equally probably variants of the orientation relationship observed within Ti-doped sapphire $[14,20,21]$ and those of Daneu et al. [22] within naturally occurring twinned rutile. No evidence for rutile twinning was observed from EBSD or TEM diffraction data. The lack of twinning is attributed to a reduced driving force for coherency, as the precipitates form at grain boundaries, where most interfaces with the corundum matrix, will not be coherent.

\subsection{Precipitation Mechanism for rutile}

It appears that rutile precipitation is not preceded by the formation of $\alpha-\mathrm{TiO}_{2}$ in the coating systems studied as no evidence for this phase was observed either in either the TEM or the XRD investigations. Neither has any ternary Ti-Al-O compound been observed in the samples examined. This is supported by the findings of Slepetys et al. [24] and Li et al. [36], 
the latter using $\mathrm{Ti}-\mathrm{Al}_{2} \mathrm{O}_{3}$ diffusion couples, who have predicted that corundum and rutile are the stable oxide phases to oxygen partial pressures below $-17 \log ($ bar $)$ at $1100^{\circ} \mathrm{C}$. This is approximately $2 / 3$ of the logarithm of the dissociation pressure of corundum at this temperature. For precipitation within the scale, oxygen partial pressure forms a boundary, below which Ti exists in some other form. For an outward flux of Ti along alumina grain boundaries, the critical partial pressure of oxygen for titania to form will therefore be some distance away from the scale/metal interface and this is clearly shown in figure 8 . Where the scale is non-protective (i.e. contains pores or cracks) the nucleation of rutile may well occur at the scale/metal interface, which could explain the location of Ti 'clusters' in figure 5.

Other possible precursors to rutile exist, which are stable below the oxygen dissociation pressure of $\mathrm{TiO}_{2}$. Titanium monoxide forms close to the dissociation pressure of oxygen for corundum at $1100^{\circ} \mathrm{C}[35]$. However the transformation sequence to $\mathrm{TiO}_{2}$ would be unlikely to result in an orientation relationship with the corundum matrix. Titanium nitride is observed to form as inclusion precipitates during casting in a turbine disk alloy, IN718. These particles then act as nucleation sites for carbides [37]. However EDS maps taken over regions similar to that shown in figure 5 have not been found to contain any regions rich in both $\mathrm{Ti}$ and $\mathrm{N}$. The direct nucleation of rutile at a grain boundary within thermally grown alumina, is substantiated by the orientation relationship observed between adjacent grains of rutile and corundum. As close to one fifth of all neighbours exist with the orientation relationship that is adopted when rutile precipitates within sapphire $[14,20,21]$. The location of the rutile precipitates within the scale is determined by the difference in oxygen dissociation pressure with that of corundum, assuming that the activities of the metal ions are not substantially different from each other. There is a reduced driving force for coherency with the sapphire matrix in these complex polycrystalline coatings that alters precipitate morphology, negates the need for twinning and destabilizes $\alpha-\mathrm{TiO}_{2}$. 


\section{Conclusions}

Grain Boundary precipitation of rutile was found to occur within alumina scales formed upon aluminized CMSX-4. Pt-aluminide and diffused-Pt coated specimens formed much less rutile. A methodology for using Rodrigues-Frank space to represent misorientation between neighbouring rutile and corundum grains has been presented. The neighbours were found to cluster about a misorientation consistent with the $<0001>_{\mathrm{c}}\{11 \overline{2} 0\}_{\mathrm{c}} / /<010>_{\mathrm{R}}\{101\}_{\mathrm{R}}$ orientation relationship that is observed within Ti doped sapphire. This is sufficient to explain the nucleation of rutile from a single corundum grain, at multi-grain junctions within the scale.

WDS and GD-OES measurements indicate Pt increases solute diffusion within $\beta$-NiAl and $\gamma-\mathrm{Ni}+\gamma^{\prime}-\mathrm{Ni}_{3} \mathrm{Al}$ coating phases. Consequently these solute species, including $\mathrm{Ti}$, can migrate to the surface more rapidly during high temperature exposure.

\section{Acknowledgements}

The authors acknowledge funding under the Rolls-Royce plc and EPSRC under the Rolls-Royce / EPSRC Strategic Partnership (EP/M005607/1 \& EP/H022309/1). This study was also supported by Nanotechnology Platform Project (NIMS Nanofabrication Platform) sponsored by the Ministry of Education, Culture, Sports, Science and Technology (MEXT), Japan. Requests for access to the underlying research data should be directed to the corresponding author and will be considered against commercial interests and data protection.

\section{Figure Captions}

Figure 1. Backscattered SEM micrographs of initial (a,b,c) microstructures and after 100 hours isothermal oxidation at $1100^{\circ} \mathrm{C}(\mathrm{d}, \mathrm{e}, \mathrm{f})$ for (left to right) aluminide, Pt-aluminide and diffused Pt coated samples.

Figure 2. Diffusion profiles obtained using WDS for major elements through initial (a,c,e) microstructures and after 100 hours isothermal oxidation at $1100^{\circ} \mathrm{C}(\mathrm{b}, \mathrm{d}, \mathrm{f})$ for (top to bottom) 
aluminide, Pt-aluminide and diffused Pt coated samples. GD-OES data is also shown in figure $2 \mathrm{e}$.

Figure 3. XRD traces taken for samples of isothermally (a, c, e) and cyclically (b, d, f) oxidized specimens at $1100^{\circ} \mathrm{C}$ for all the time periods tested. Indexed peaks are shown by the following abreviations: $\beta-\mathrm{Ni}(\mathrm{Pt}) \mathrm{Al}, \alpha-\mathrm{Al}_{2} \mathrm{O}_{3}, \gamma^{\prime}-\mathrm{Ni}_{3} \mathrm{Al}, \gamma-\mathrm{Ni}(\mathrm{Pt}) \mathrm{S}-\mathrm{NiAl}_{2} \mathrm{O}_{4}, \mathrm{R}-\mathrm{TiO}_{2}, \xi-\mathrm{PtAl}_{2}$.

Figure 4. Diffusion profiles obtained using WDS for minor elements through initial (a, c, e) microstructures and after 100 hours isothermal oxidation at $1100^{\circ} \mathrm{C}(\mathrm{b}, \mathrm{d}, \mathrm{f})$ for (top to bottom) aluminide, Pt-aluminide and diffused Pt coated samples. GD-OES data is also shown in figure 4e.

Figure 5. Elemental maps obtained using WDS for $\mathrm{Al}$ and Ti within (top) aluminide, (middle) Pt-aluminide and (bottom) diffused Pt coated samples after 100 hours at $1100^{\circ} \mathrm{C}$.

Figure 6. Brightfield image obtained using STEM mode of TGO surface (top left) and corresponding EDS maps for diffused Pt coated sample after 25 hours at $1100^{\circ} \mathrm{C}$.

Figure 7. Brightfield image obtained using STEM mode (a) with corresponding orientation map (b) revealing the rutile $\mathrm{TiO}_{2}$ and upper $\left(\mathrm{Al}_{2} \mathrm{O}_{3} \mathrm{u}\right)$ and lower $\left(\mathrm{Al}_{2} \mathrm{O}_{3} 1\right)$ alumina grains. Inverse pole figures of fundamental zones for both tetragonal $(4 / \mathrm{mmm})(\mathrm{c})$ and trigonal $(\overline{3} \mathrm{~m})$ (d) structures also shown with the orientations of each grain circled.

Figure 8. Maps obtained using EBSD containing indexed corundum and rutile grains, which were used for nearest neighbor disorientation analysis. Simultaneous EDS data is also shown. 
Figure 9. Rodrigues-Frank vectors within RF space for neighboring corundum (a) and rutile grains (b) extracted from EBSD data. Disorientation vectors for rutile-corundum neighbors (c). Corresponding disorientation angle distribution (d).

Figure 10. Orientations of corundum (a) and rutile (b) that give rise to an orientation matrix of unity within the 'HKL' hexagonal axes description. The corundum structure is rotated by $90^{\circ}$ about the $[0,1,0]$ Cartesian axis, bringing the $[\overline{2} 110]$ and $[011]$ plane normals close to parallel as depicted in (c).

\section{Table Captions}

Table 1. Rodrigues-Frank vectors that give rise to the $<0001>_{C}\{11 \overline{2} 0\}_{C} / /<010>_{R}\{101\}_{R}$ misorientation for rutile and corundum and lie within the appropriate RF misorientation space.

\section{References}

[1] Hayashi S, Wang W, Sordelet D, Gleeson B. Metall Mater Trans A 2005;36:1769.

[2] Zhang Y, Pint BA, Haynes JA, Wright IG. Surf Coat Technol 2005;200:1259.

[3] Wu RT, Kawagishi K, Harada H, Reed RC. Acta Mater 2008;56:3622.

[4] Wu RT, Wang X, Atkinson A. Acta Mater 2010;58:5578.

[5] Pint BA, Haynes JA, Zhang Y. Surf Coat Technol 2010;205:1236.

[6] Al-Hadhrami LM, Tawancy HM. J Eng Gas Turb Power 2011;133:1.

[7] Marino KA, Carter EA. Acta Mater 2010;58:2726.

[8] Marino KA, Carter EA. Acta Mater 2008;56:3502.

[9] Hanaor DAH, Sorrell CC. J Mater 2010;46:855.

[10] Kumar KNP. Scripta Metall Mater 1995;32:873.

[11] Ding XZ, Liu XH, He YZ. J Mater Sci Lett 1996;15:1789. 
[12] Meng D, Wu X, Fan X, Zhang Z, Chen H et al. Acta Geol Sin (Eng. Ed.) $2008 ; 82: 371$.

[13] Sridharan S, Xie L, Jordan EH, Gell M. Surf Coat Technol 2004;179:286.

[14] Xiao SQ, Dahmen U, \& Heuer AH. Phil Mag A 1997;75:221.

[15] Petot-Ervas G, Saadi B, Petot C, Loudjani M. J Eur Ceram Soc 1996;17:943.

[16] Atkinson KJW, Grimes RW, Levy MR, Coull ZL, English T. J Eur Ceram Soc $2003 ; 23: 3059$.

[17] Grimes RW. J Am Ceram Soc 1994;77:378.

[18] Mckee DW, Aleshin E. J Am Ceram Soc 1963;46:54.

[19] Roy SK, Coble RL. J Am Ceram Soc 1968;51:1.

[20] Phillips DS, Heuer AH, Mitchell TE. Phil Mag A 1980;42:385.

[21] He J, Lagerlof KPD, Heuer AH. J Am Ceram Soc 2011;94:1272.

[22] Daneu N, Recnik, A, \& Mader W. Am Mineral 2014;99:612.

[23] Langensiepen RE, Tressler RE, Howell PR. J Mat Sci 1983;18:2771.

[24] Slepetys RA, Vaughan PA. J Phys Chem 1969;73:2157.

[25] Swadźba R, Hetmańczyk M, Wiedermann J, Swadźba L, Moskal G, et al. Surf Coat Technol 2013;215:16.

[26] Shirvani K, Firouzi S, Rashidghamat A. Corros Sci 2012;55:378.

[27] Das DK. Prog Mater Sci 2013;58:151.

[28] Rauch EF, portillo J, Nicolopoulos S, Bultreys D, Rouvimov S, Moeck P. Ziets Krist 2010;225:103.

[29] Bachmann F, Hielscher R, Schaeben H. Sol St Phen 2010;160:63.

[30] Heinz A, Neumann P. Acta Crystallogr A 1991;47:780.

[31] He Y, Jonas JJ. J Appl Cryst 2007;40:559.

[32] Zhang Y, Haynes JA, Pint BA, Wright IG, Lee WY. Surf Coat Technol 2003;163164:19-24.

[33] Chen MW, Glynn ML, Ott RT, Hufnagel TC, Hemker KJ. Acta Mater 2003;51:4279. 
[34] Morawiec, A. J Appl Cryst 1995;28;289.

[35] Morawiec, A. Acta Crystallogr A 1997;53;273.

[36] Li XL, Hillel R, Teyssandier F, Choi SK, Van Loo FJJ. Acta Metall Mater $1992 ; 40: 3149$.

[37] Mitchell A. In: Advanced materials and processing techniques for structural applications; (ASM) 1987. p. 233. 